Portland State University

PDXScholar

\title{
Mathematical Modeling of Vaccinations: Modified SIR Model, Vaccination Effects, and Herd Immunity
}

Tina Huyen Luong

Portland State University

Follow this and additional works at: https://pdxscholar.library.pdx.edu/honorstheses

Let us know how access to this document benefits you.

\section{Recommended Citation}

Luong, Tina Huyen, "Mathematical Modeling of Vaccinations: Modified SIR Model, Vaccination Effects, and Herd Immunity" (2019). University Honors Theses. Paper 695.

https://doi.org/10.15760/honors.712

This Thesis is brought to you for free and open access. It has been accepted for inclusion in University Honors Theses by an authorized administrator of PDXScholar. Please contact us if we can make this document more accessible: pdxscholar@pdx.edu. 
Portland State University

PDXScholar

University Honors Theses

University Honors College

May 25th, 2019

\section{Mathematical Modeling of Vaccinations: Modified SIR Model, Vaccination Effects, and Herd Immunity}

Tina Huyền Lương

Portland State University

Under the supervision of Prof. Jeffrey Ovall

Let us know how access to this document benefits you.

Follow this and additional works at: https://pdxscholar.library.pdx.edu/honorstheses

Recommended Citation

Luong, Tina H., "Mathematical Modeling of Vaccinations: Modified SIR Model, Vaccination Effects, and Herd Immunity" (2019).

University Honors Theses. Paper 695.

10.15760/honors.712

This Dissertation/Thesis is brought to you for free and open access. It has been accepted for inclusion in University Honors Theses by an authorized administrator of PDXScholar. For more information, please contact pdxscholar@pdx.edu. 


\title{
Mathematical modeling of vaccinations: modified SIR model, vaccination effects, and herd immunity
}

\author{
Tina Huyền Lương \\ Portland State University
}

\begin{abstract}
Despite the clear effects and benefits of vaccinations on a population, there are many individuals that choose to not vaccinate for non-medical reasons, giving rise to anti-vaccination movements and vaccine hesitancy. This paper introduces the different types of vaccines and the effects of vaccines in the body and provides an examination of a case study of the 2019 Pacific Northwest measles outbreak. The outbreak is modeled using a proposed modified SIR model and solved using the Fourth-Order Runge-Kutta method. The results suggest that around day 20, almost the entire population becomes infected with the vaccine-resistant strain, which outcompetes the wild-type strain, and the vaccination rate of Clark and Multnomah county suggest that the herd immunity effect does not occur. Many limitations exist for the proposed modified SIR model, with two major limitations being the lack of spatial consideration and the assumption of homogeneous mixing of the population.
\end{abstract}

\section{INTRODUCTION}

Before vaccinations in the 20th century, there have been over half a million cases of measles, over 150,000 cases of mumps, and around 50,000 cases of smallpox annually, and since the introduction of vaccines, the number of cases for measles, mumps, and smallpox decreased by $99.9 \%, 98.7 \%$, and $100 \%$ as of 2009 , respectively. ${ }^{1}$ Time and time again, vaccines have displayed their ability to eradicate diseases and decrease disease occurrences; however, many individuals still find themselves hesitant of vaccinations due to many factors such as vaccine controversy, cultural/religious/personal beliefs, past experience, family history, personal feelings of control, community and peer influence, and so forth. To observe the effects of vaccination and herd immunity on the number of disease occurrences in a population, an examination of a case study of the 2019 Pacific Northwest measles outbreak is conducted, and the outbreak is modeled by a modified SIR model.

\section{VACCINATION: TYPES AND EFFECTS}

\section{Types of vaccines}

Many different types of vaccines have been developed to address different concerns and conditions, such as 
immune response, infection dynamics, environment, and so forth. The most commonly seen vaccines are live, attenuated, inactivated, subunit, conjugate, and toxoid vaccines.

1) Live, attenuated vaccines are composed of a weakened version of the live, infectious agent. e.g. MMR vaccine (measles, mumps, rubella), varicella vaccine (chickenpox)

2) Inactivated vaccines are composed of the dead infectious agent. e.g. inactivated poliovirus vaccine, flu shot, hepatitis A vaccine

3) Subunit and conjugate vaccines include only parts of the infectious agent (like its antigen, protein, sugar, etc.), rather than the whole microbe. ${ }^{2}$

e.g. part of DTaP vaccine (whooping cough), Hib (Haemophilus influenzae type B) vaccine

4) Toxoid vaccines are composed of the weakened version of the toxins that certain bacteria release. Toxins are weakened to the point where they cannot cause illness. ${ }^{2}$ e.g. tetanus, diphtheria

\section{The intersections of the body's immune response and vaccinations}

To better understand how vaccines work, one would first need to recognize the ways in which the human body tackles illness and builds immunity. An over-generalized explanation is given below.

Infectious agents, such as bacteria or viruses, invade the body and replicate, causing an infection and leading to an illness. To begin, immune cells rush to the infected area and induce inflammation, while macrophages and neutrophils engulf the infectious agents through endocytosis, digest them using enzymes, present their antigens on the cell surface, and secrete signaling molecules to recruit more immune cells. When the infectious agent is a virus, infected host cells secrete interferons (special proteins) that signal uninfected, neighboring cells to release substances that inhibit the reproduction of viruses and decrease the permeability of cells. ${ }^{3}$ T-cells and B-cells are also present and attempting to attack infected cells and infectious agents, respectively.

As all this is occurring, dendritic cells collect antigens from the infectious agents, display them on its surface, and initiate the body's adaptive immune response by presenting the antigens to naive T-cells in secondary lymphoid organs. ${ }^{4}$ The exposed naive T-cells then replicate to produce a variety of T-cells, including memory and helper T-cells, and helper T-cells go on to activate naive B-cells. B-cells reproduce to create memory Bcells, which remember the appropriate antibody needed for the specific antigen of an infectious agent and remain in the body after infection, and plasma cells, which are responsible for releasing an influx of antibodies. ${ }^{3}$ T-cells are a part of the body's cell-mediated immune response and target infected host cells, whereas B-cells directly target the infectious agent and release antibodies to combat antigens on the agent's surface and any free-flowing antigens in the blood or lymph. ${ }^{3}$

Though seemingly efficient, it typically takes around seven to ten days after the exposure to the antigen for Bcells to respond with the appropriate antibody because each B-cell creates only one specific configuration of the antibody and antigen receptor, corresponding to only one specific antigen. However, depending on the illness, seven to ten days may be too late for the body to fight off the illness alone or for the individual to not develop complications. Since memory B-cells remain in the body and await re-exposure to the antigen, the development of memory B-cells serves as the goal of vaccinations. Vaccines work to help an individual build 
immunity by imitating an infection. From the pseudo-infection, the body undergoes its immune response, producing memory $\mathrm{T}$ - and $\mathrm{B}$-cells in the process. In the event of a true infection, the immune system views this as a repeated encounter, and the memory B-cells rapidly produce a surge of the appropriate, corresponding antibodies to target the antigens of the infectious agents. This allows the individual to skip the week-long wait needed for B-cells to frantically find the perfect match for the antigens presented.

\section{HERD IMMUNITY}

While vaccines can effectively prevent illness, not everyone can become vaccinated due to age, illness, allergy, compromised immune system, etc. Nevertheless, unvaccinated individuals can benefit from the immunity of vaccinated individuals via herd immunity. Herd immunity is a form of indirect protection for susceptible individuals in a population where having enough vaccinated individuals in the population decrease the number of susceptible hosts for an infectious agent, thus preventing or limiting the spread of disease. If cases of the disease do occur, they are often easier to isolate or contain.

\section{PROPOSED MODIFIED SIR MODEL}

Many models have been produced to predict the spread of infectious disease and demonstrate possible outcomes given a set of circumstances. Most generally known, the SIR model involves the examination of the number of people with respect to time in three defined categories: susceptible (S), infected (I), and recovered (R). Dr. Angela McLean and Dr. Almut Scherer introduced the following system of differential equations for a modified SIR model, accounting for the existence of vaccine-resistant strains of infectious agents and the addition of a category for vaccinated individuals (V).

\section{Equations for Modified SIR Model ${ }^{5}$}

$$
\begin{aligned}
& \frac{d V}{d t}=p \mu N-\beta_{w}\left(1-\varphi_{w}\right) V I_{w}-\beta_{r}\left(1-\varphi_{r}\right) V I_{r}-\mu V \\
& \frac{d S}{d t}=(1-p) \mu N-(1-Q) \beta_{w} S I_{w}-Q \beta_{w} S I_{w}-\beta_{r} S I_{r}-\mu S \\
& \frac{d I_{w}}{d t}=(1-Q) \beta_{w} S I_{w}+\beta_{w}\left(1-\varphi_{w}\right) V I_{w}-\gamma I_{w}-\mu I_{w} \\
& \frac{d I_{r}}{d t}=Q \beta_{w} S I_{w}+\beta_{r} S I_{r}+\beta_{r}\left(1-\varphi_{r}\right) V I_{r}-\gamma I_{r}-\mu I_{r} \\
& \frac{d R}{d t}=\gamma I_{w}+\gamma I_{r}-\mu R
\end{aligned}
$$

where the meanings of the above parameters and variables are displayed in Table 1 below. 
Table 1. Definition of parameters and variables for modified SIR model differential equations. ${ }^{5}$

\begin{tabular}{cl}
\hline Parameter/Variable & Meaning \\
\hline$p$ & Fraction of individuals vaccinated at birth \\
$\mu$ and $\gamma$ & Death and recovery rate, respectively \\
$\beta_{w}$ and $\beta_{r}$ & Transmission/effective-contact rate for wild-type strain; for vaccine-resistant strain \\
$\varphi_{w}$ and $\varphi_{r}$ & Fraction of infections with wild-type strain; with vaccine-resistant strain \\
$Q$ & Rate of mutation/mutation probability \\
$V(t)$ & Number of vaccinated individuals at a given time \\
$S(t)$ & Number of susceptible individuals at a given time \\
$I_{w}(t)$ & Number of infected individuals with wild-type strain at a given time \\
$I_{r}(t)$ & Number of infected individuals with vaccine-resistant strain at a given time \\
$R(t)$ & Number of recovered/permanently immune individuals at a given time \\
$N(t)$ & Total population (sum of $V(t), S(t), I_{w}(t), I_{r}(t)$, and $\left.R(t)\right)$ \\
\hline
\end{tabular}

The proposed modified SIR model assumes the following:

- Immunity gained from infection of either strain (vaccine-resistant or wild-type) grants life-long immunity against all strains for the individual.

- Susceptible and vaccinated individuals can be infected by either strain, thus taking into consideration the waning immunity of vaccines.

- With enough cases, the vaccine-resistant strain is not at a selective disadvantage and takes over as the primary strain infecting individuals.

- General assumptions of SIR models hold (population mixes homogeneously, only way to leave susceptible category is by becoming infected, etc.).

\section{CASE STUDY: 2019 PACIFIC NORTHWEST MEASLES OUTBREAK}

On January 3rd, 2019, the first case of measles was confirmed in a child that returned from a trip to Ukraine, and the measles outbreak began in Clark county, Washington. ${ }^{6}$ By January 18th, 2019, the Clark County Council Chair, Eileen Quiring, declared a public health emergency due to the measles outbreak, and Governor Jay Inslee declared a state of emergency in Washington on January 25th after the number of measles cases reached 25 with 12 suspected cases pending. ${ }^{67}$ There have been 53 exposure sites identified, ranging from health care facilities, schools, and childcare centers to workplaces, retail stores, and other public places. ${ }^{6}$ As of April 29th, 2019, Clark County Public Health declared that the measles outbreak has concluded. ${ }^{6}$

Now, this case study will focus specifically on the cases examined in Clark county, Washington and Multnomah county, Oregon. Using the data available on April 29th, 2019, Clark and Multnomah county has 65 cases in which the affected individuals are unvaccinated, three cases in which the affected individuals 
received one dose of the MMR vaccine, and seven cases in which the immunization status is unverified. ${ }^{6,8}$ Some critical assumptions are made for this study:

- The unverified cases are assumed to be unvaccinated, thus bringing the total unvaccinated cases to 72 and vaccinated cases to three.

- At time $t=0$, let the 72 unvaccinated cases and three vaccinated cases all be developed at the same time.

- Suppose the vaccinated cases are cases in which the individuals are infected with the vaccine-resistant strain and the unvaccinated cases are cases in which the individuals are infected with the wild-type strain.

\section{Setting: defining parameters and initial values}

As of July 1st, 2018, the populations of Clark and Multnomah county are 481,857 people and 811,880 people, with a vaccination rate of $0.81 \%$ and $0.87 \%$, respectively, for one dose of MMR. ${ }^{9.11}$ Assuming all vaccinated individuals receive the first dose of MMR at birth, the fraction of vaccinated individuals at birth $p$ is

$$
p=\frac{v_{m} N_{m}+v_{c} N_{c}}{N_{m}+N_{c}}=\frac{0.87(811,880)+0.81(481,857)}{811,880+481,857}=8.47653 \times 10^{-1}
$$

where $v_{m}, v_{c}$ are the vaccination rates and $N_{m}, N_{c}$ are the populations for Multnomah and Clark county. The death rate for measles $\mu$ is two out of a thousand deaths per year, or $5.47945 \times 10^{-6}$ per day. ${ }^{12}$ The recovery rate for measles $\gamma$ is $5.0 \times 10^{-2}$ per day, the reciprocal of the duration of infection, which is approximately twenty days. ${ }^{13}$

Assuming the effective contact rate for the wild-type strain $\beta_{w}$ and vaccine-resistant strain $\beta_{r}$ are the same, the rate is obtained from the equation below.

$$
\beta_{w}=\beta_{r}=\frac{R_{0} \gamma}{N_{\text {total }}}=\frac{(15)(0.05)}{1,293,737}=5.79716 \times 10^{-7} \text { transmissions } / \text { contact }
$$

where $R_{0}$ is the basic reproduction number for measles, $\gamma$ is the recovery rate, and $N_{\text {total }}$ is the combined population of Multnomah and Clark county. For measles, the basic reproduction number is between 12-18, and the average of the two values was chosen for the calculation. ${ }^{14}$ Furthermore, the probability of the measles virus mutating $Q$ is approximately $1.2 \times 10^{-4}$ mutations per replication. ${ }^{15}$

Using the data obtained from the 2019 Pacific Northwest measles outbreak as of April 29th, 2019 and supposing the unconfirmed cases are unvaccinated cases, there are a total of 72 unvaccinated cases and three vaccinated cases, where the person received one dose of $\mathrm{MMR}^{6,8}$ Let the three vaccinated individuals be infected by the vaccine-resistant strain. Then, the fraction of individuals infected by the wild-type strain $\varphi_{w}$ and fraction of individuals infected with the vaccine-resistant strain $\varphi_{r}$ are 


$$
\varphi_{w}=\frac{\text { unvaccinated cases }}{\text { total cases }}=\frac{72}{72+3}=9.6 \times 10^{-1} \quad \text { and } \quad \varphi_{r}=\frac{\text { vaccinated cases }}{\text { total cases }}=\frac{3}{72+3}=4.0 \times 10^{-2}
$$

Now, the initial state for each category will be based on the data from the 2019 Pacific Northwest measles outbreak dated April 29th, 2019. From the data, the initial number of infected individuals with the wild-type strain $I_{w}(0)$ and the vaccine-resistant strain $I_{r}(0)$ are 72 people and three people, respectively. The initial value for the number of vaccinated individuals is calculated by multiplying the total population $N_{\text {total }}$ by the fraction of vaccinated individuals at birth $p$ and subtracting the initial number of infected individuals with the vaccine-resistant strain. That is,

$$
V(0)=p \times N_{\text {total }}-I_{r}(0)=\left(8.47653 \times 10^{-1}\right)(1,293,737)-3=1.096637 \times 10^{6} \text { people }
$$

It follows that the initial number of susceptible individuals $S(0)$ is

$$
S(0)=N_{\text {total }}-V(0)-I_{r}(0)-I_{w}(0)=1.97025 \times 10^{5} \text { people }
$$

and the initial number of recovered individuals $R(0)$ is zero. All the calculated values above for each parameter and initial value is displayed in the table below.

Table 2. Value given to each parameter and initial state ${ }^{1218,15}$

\begin{tabular}{ccc}
\hline Parameter & Value & Units \\
\hline$p$ & $8.47653 \times 10^{-1}$ & - \\
$\mu$ & $5.47945 \times 10^{-6}$ & day $^{-1}$ \\
$\gamma$ & $5.0 \times 10^{-2}$ & day $^{-1}$ \\
$\beta_{w}$ and $\beta_{r}$ & $5.79716 \times 10^{-7}$ & transmissions $/$ contact \\
$\varphi_{w}$ and $\varphi_{r}$ & $9.6 \times 10^{-1}$ and $4.0 \times 10^{-2}$ & - \\
$Q$ & $1.2 \times 10^{-4}$ & mutations/replication \\
$V(0)$ & $1.096637 \times 10^{6}$ & people \\
$S(0)$ & $1.97025 \times 10^{5}$ & people \\
$I_{w}(0)$ & 72 & people \\
$I_{r}(0)$ & 3 & people \\
$R(0)$ & 0 & people \\
\hline
\end{tabular}

\section{Solving the Modified SIR Model using the Fourth-Order Runge-Kutta Method}

The Fourth-Order Runge-Kutta method is a method used to find an approximate solution of initial value problems of the form $\frac{d y(t)}{d t}=f(t, y(t))$ with $y\left(t_{0}\right)=y_{0}$, where $y$ can be a vector-valued function. For this case study, 


$$
y(t)=\left[\begin{array}{c}
V(t) \\
S(t) \\
I_{w}(t) \\
I_{r}(t) \\
R(t)
\end{array}\right]
$$

and

$$
f(t, y(t))=\left[\begin{array}{c}
p \mu N-\beta_{w}\left(1-\varphi_{w}\right) V I_{w}-\beta_{r}\left(1-\varphi_{r}\right) V I_{r}-\mu V \\
(1-p) \mu N-(1-Q) \beta_{w} S I_{w}-Q \beta_{w} S I_{w}-\beta_{r} S I_{r}-\mu S \\
(1-Q) \beta_{w} S I_{w}+\beta_{w}\left(1-\varphi_{w}\right) V I_{w}-\gamma I_{w}-\mu I_{w} \\
Q \beta_{w} S I_{w}+\beta_{r} S I_{r}+\beta_{r}\left(1-\varphi_{r}\right) V I_{r}-\gamma I_{r}-\mu I_{r} \\
\gamma I_{w}+\gamma I_{r}-\mu R
\end{array}\right]
$$

This method involves finding four approximations of slopes $K_{i}, 1 \leq i \leq 4$, for each of the population functions per time step: one at the beginning, two at the midpoint, and one at the end of the time step. The Fourth-Order Runge-Kutta method generates an approximation $y_{k}$ of the true state of the system $y\left(t_{k}\right)$ at a time $t_{0} \leq t_{k} \leq T$ through an iteration and is given by

$$
y_{k+1}=y_{k}+\frac{h}{6}\left(K_{1}+2 K_{2}+2 K_{3}+K_{4}\right)
$$

such that

$$
\begin{gathered}
K_{1}=f\left(t_{k}, y_{k}\right) \\
K_{2}=f\left(t_{k}+\frac{h}{2}, y_{k}+\frac{h}{2} K_{1}\right) \\
K_{3}=f\left(t_{k}+\frac{h}{2}, y_{k}+\frac{h}{2} K_{2}\right) \\
K_{4}=f\left(t_{k}+h, y_{k}+h K_{3}\right)
\end{gathered}
$$

$0<h<1$ is the step size chosen and $t_{k}=t_{0}+k h, k \geq 0 .{ }^{16}$

For the Runge-Kutta method to be of fourth order, the worst approximation of the true state of the system is

$$
\max _{0 \leq \mathrm{t}_{\mathrm{k}} \leq \mathrm{T}}\left|y\left(t_{k}\right)-y_{k}\right|<C h^{4}
$$

and decaying by a rate of $h^{4}$, with $C$ as a constant. ${ }^{16}$

Now, given the system of differential equations in the modified SIR model proposed, the Fourth-Order Runge-Kutta method was used to approximate the solution of the system from the time $t=0$ to 100 days. The following code was ran on GNU Octave using a step size $h$ of 0.01 and the defined values given in Table 2 . 


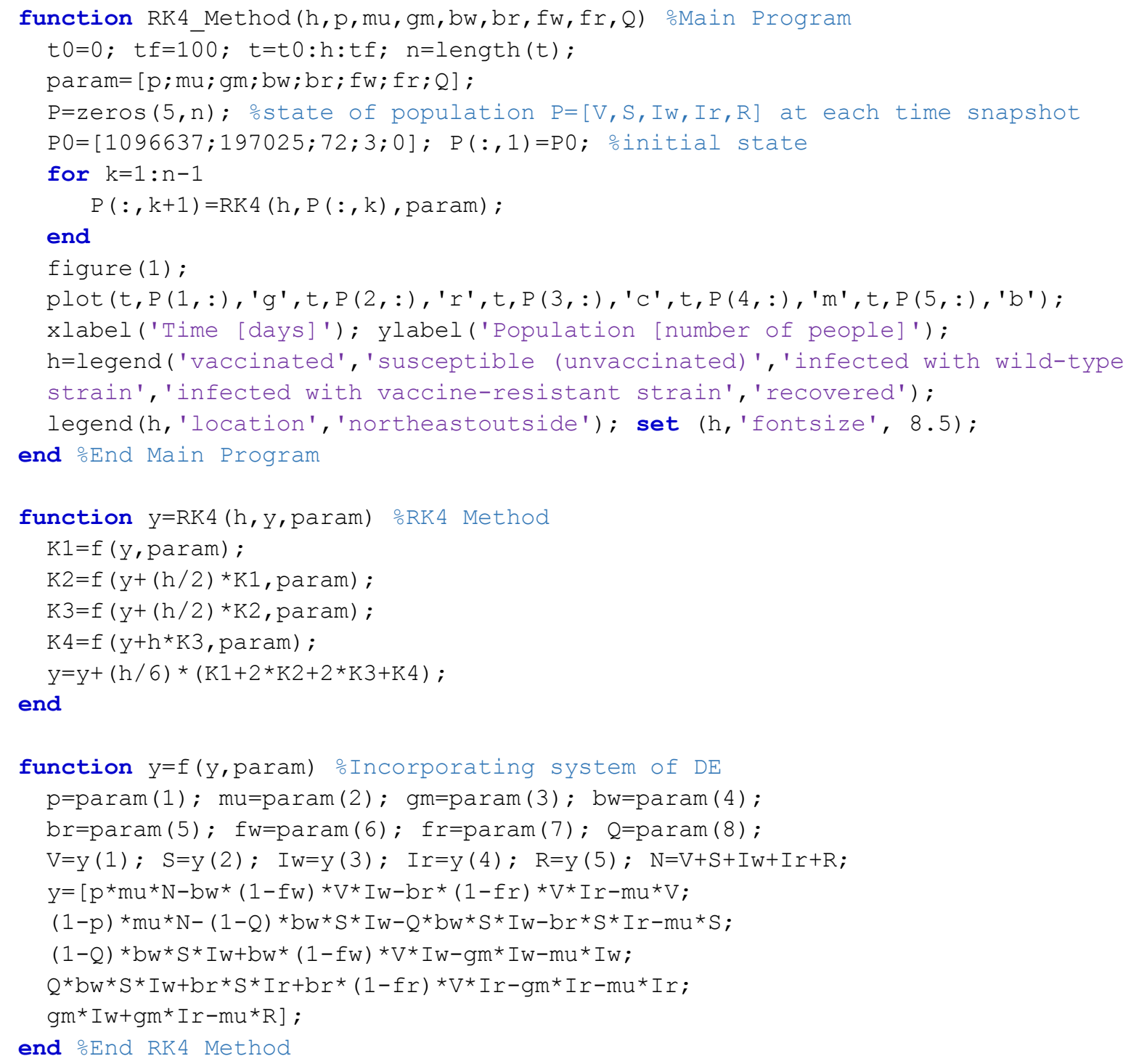

\section{Results}

Solving the system of differential equations using the Fourth-Order Runge-Kutta method and the code above gave the following figure. Figure 1 displays five curves, with each representing the approximation for the number of vaccinated, susceptible, infected with wild-type strain, infected with vaccine-resistant strain, or recovered individuals in the combined population of Clark and Multnomah county on any day from zero to 100 days. These approximations for the different population functions suggest that around day 20, almost the entire population becomes infected with the vaccine-resistant strain. With a large vaccinated population initially, the vaccine-resistant strain outcompetes the wild-type strain, rendering the vaccines for measles ineffective. In the case of a large unvaccinated population, the wild-type strain outcompetes the vaccineresistant strain, as indicated in Figure 2, and the entire population becomes infected as well. Based on the 
results, a vaccination rate of approximately $96 \%$ was not enough to benefit from herd immunity effects and contain the measles outbreak, especially since a vaccine-resistant strain was introduced into the population.

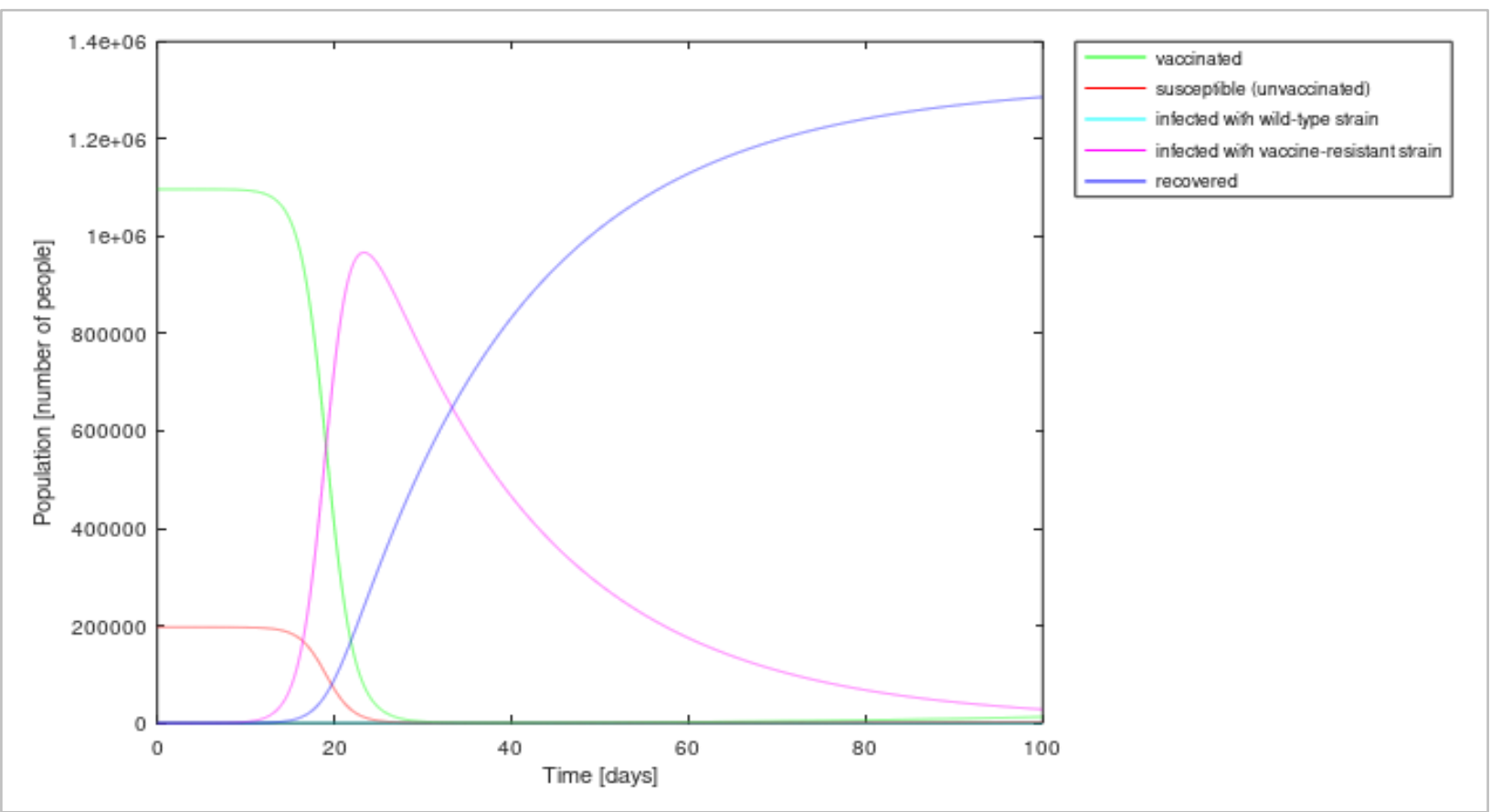

Figure 1. RK4 approximation of the number of vaccinated, unvaccinated, infected, and recovered individuals in the combined population of Clark and Multnomah county after measles exposure.

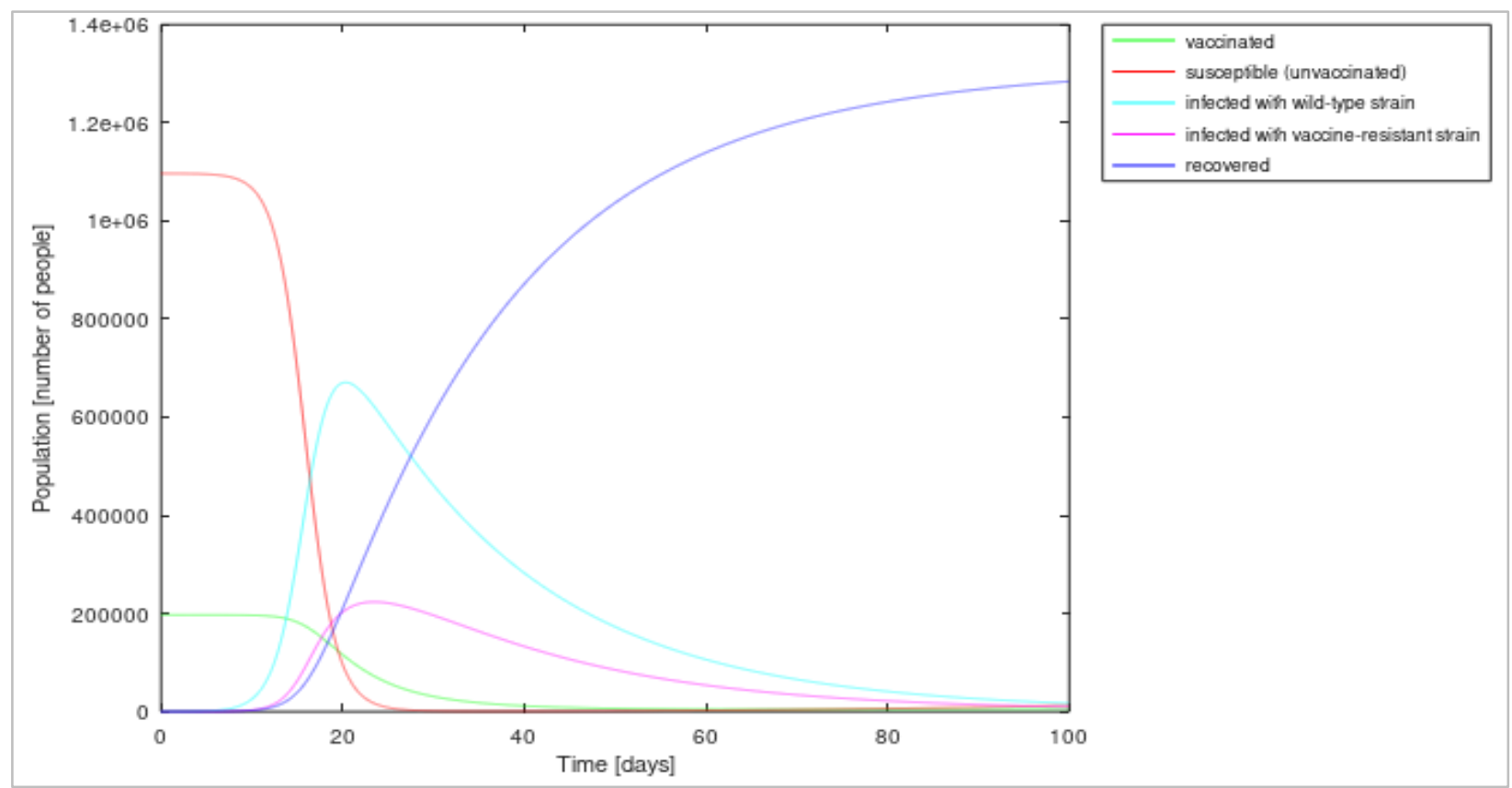

Figure 2. RK4 Approximation of modified SIR model solution given initial unvaccinated population $V(0)=$ $1,096,637$ people, initial vaccinated population $S(0)=197,025$ people, and fraction of population at vaccinated at birth $p=0.152347$. 


\section{CONCLUSION}

While the death rate for measles is low, two deaths per 1,000 cases, $30 \%$ of measles cases have reported having one or more complications, as measles suppresses an infected individual's immune system and causes them to become prone to infection. ${ }^{17}$ More common, minor complications are diarrhea and ear infections, which affect one out of ten cases, though the ear infections can fester and lead to permanent hearing loss. ${ }^{18}$ Severe complications due to measles consist of pneumonia, affecting one out of 20 cases, and encephalitis, affecting one of 1,000 cases. ${ }^{18}$ Pneumonia is the most common cause of death in young children affected by measles, and encephalitis (brain swelling) leads to convulsions and can cause deafness or neurological damage. ${ }^{18}$ Before vaccinations in the 20th century, there have been over half a million cases of measles, and since the introduction of vaccines, the number of cases for measles decreased by $99.9 \%{ }^{1}$

Mathematical models can help with creating visual representation and predicting long-term behavior, but there are many limitations to using them. For instance, the modified SIR model used to model the effects of measles on a population contained many assumptions such as homogeneous mixing of the population, no birth and migration, no spatial consideration, and so forth. Homogeneous mixing of the population resulted in there being more infected individuals than what would likely happen in a realistic scenario. In real situations, the population does not mix homogeneously, and cases of infection are isolated to prevent further spread of disease. Having a large vaccinated population and a vaccine-resistant strain of measles introduced negated herd immunity effects and rendered the MMR vaccine ineffective in the case study. Furthermore, the model assumes that when the measles strain mutates, a vaccine-resistant strain is guaranteed to be produced, when in real situations, the probability of such a strain being formed from a mutation is low in a largely vaccinated population. Despite the limitations, mathematical models used for simulations are instructive and can be modified and advanced to reduce error and account for further variables, such as space/location.

\section{REFERENCES}

1. Vaccine Benefits. National Institute of Allergy and Infectious Diseases. https:/www.niaid.nih.gov/ research/vaccine-benefits. Published May 18, 2019. Accessed May 21, 2019.

2. Vaccine Types. National Institute of Allergy and Infectious Diseases. https://www.niaid.nih.gov/ research/vaccine-types. Published May 18, 2019. Accessed May 21, 2019.

3. Urry LR, Cain ML, Wasserman SA, Minorsky PV, Reece JB. The immune system. Campbell Biology in Focus. $2^{\text {nd }}$ ed. Pearson; 2016: 733-750.

4. Ni K, O’Neill HC. The role of dendritic cells in T cell activation. Immunology and Cell Biology. https://www.ncbi.nlm.nih.gov/pubmed/9243286. Published June 1997. Accessed March 19, 2019.

5. Scherer A, McLean AR. Mathematical models of vaccinations. British Medical Bulletin. 2002; 62(1): 187-199. https://academic.oup.com/bmb/article/62/1/187/281114. Published July 1, 2002 Accessed February 10, 2019. 
6. Measles investigation. Clark County Washington. https:/www.clark.wa.gov/public-health/measlesinvestigation. Published April 29, 2019. Accessed May 21, 2019.

7. Measles case count up to 25 confirmed, 12 suspect. Clark County Washington. https://www.clark.wa. gov/public-health/measles-case-count-25-confirmed-12-suspect. Published January 24, 2019. Accessed May 21, 2019.

8. Measles Outbreaks 2019. Multnomah County. https://multco.us/measles2019. Published April 24, 2019. Accessed May 21, 2019.

9. U.S. Census Bureau QuickFacts: Clark County, Washington; Multnomah County, Oregon. Census Bureau QuickFacts. https:/www.census.gov/quickfacts/fact/table/clarkcountywashington,multnomah countyoregon/PST045218. Accessed May 21, 2019.

10. What are the immunization rates for children in Clark County? Clark County Washington. https://www. clark.wa.gov/public-health/faq/what-are-immunization-rates-children-clark-county. Accessed May 21, 2019.

11. Oregon Child Immunization Rates. Oregon Health Authority: Oregon Child Immunization Rates: Vaccines and Immunization : State of Oregon. https:/www.oregon.gov/oha/PH/PREVENTION WELLNESS/VACCINESIMMUNIZATION/Pages/researchchild.aspx. Accessed May 21, 2019.

12. Measles Data and Statistics. Centers for Disease Control and Prevention. https://www.cdc.gov/measles/ downloads/measlesdataandstatsslideset.pdf

13. Immunology and Vaccine - Preventable Diseases - Pink Book - Measles. Centers for Disease Control and Prevention. https://www.cdc.gov/vaccines/pubs/pinkbook/downloads/meas.pdf

14. Guerra FM, Bolotin $S$, $\operatorname{Lim} G$, et al. The basic reproduction number $\left(\mathrm{R}_{0}\right)$ of measles: a systematic review. The Lancet Infectious diseases. 2017; 17(12): e420-e428. https://www.ncbi.nlm.nih.gov/ pubmed/28757186. Published December 2017. Accessed May 21, 2019.

15. Schrag SJ, Rota PA, Bellini WJ. Spontaneous mutation rate of measles virus: direct estimation based on mutations conferring monoclonal antibody resistance. Journal of Virology. 1999; 73(1): 51-54. https://www.ncbi.nlm.nih.gov/pmc/articles/PMC103807/. Published January 1999. Accessed May 21, 2019.

16. Kutta MW. Beitrag zur näherungsweisen integration totaler differentialgleichungen. Zeitschrift für Mathematik und Physik. 1901; 46: 435-452.

17. Epidemiology and Prevention of Vaccine-Preventable Diseases Chapter 13: Measles. Centers for Disease Control and Prevention. https://www.cdc.gov/vaccines/pubs/pinkbook/meas.html\#complicatio ns 
18. Measles (Rubeola) Complications. Centers for Disease Control and Prevention. https:/www.cdc.gov/ measles/about/complications.html 